\title{
Identification of Low Back Injury from EMG Signals Using a Neural Network Model
}

\author{
Yanfeng Hou, Jacek M. Zurada, Fellow, IEEE, Waldemar Karwowski, Member, IEEE, and William S. Marras
}

\begin{abstract}
-we propose a novel neural network model for the identification of low back injury using electromyography (EMG) data. By connecting task condition variables to the second hidden-layer of the neural network, the importance of those variables can be improved. A 4-muscle method and a 10-muscle method are discussed. A higher classification accuracy was achieved by the 10-muscle method since it takes the correlation between muscle activities into account. We also found that identification accuracy decreases when the object weight or the lifting height increases. The obtained results improve our understanding of low back disorders and provide important guidance for future experimental studies.
\end{abstract}

\section{INTRODUCTION}

Low back disorders (LBDs) are one of the most common musculoskeletal problems reported in industry which result in substantial costs to society [1][2]. Diagnoses and classification schemes of low back disorders are important because appropriate treatment can be administered and the risk of exacerbating the problem can be minimized. However, low back disorders can not be easily assessed [3]. Several traditional approaches have been discussed in [1] and [4]. In the approach identifying a pathoanatomic source of the LBDs, patients are classified according to the presumed structure that is injured or painful. However, a pathoanatomic diagnosis has been found in fewer than $15 \%$ of patients with LBDs. In the Quebec study classification system, the classification accounts for the fact that structural abnormalities are not always identifiable in LBDs and recognizes that LBDs are time dependent. However, common symptoms can arise from different structural problems, thus confounding the diagnosis.

In view of limitations and disadvantages of the above mentioned methods, we consider using EMG responses of the trunk muscles to identify cases of LBDs. EMG response is a direct reflection of muscular activity and can be used to represent the dynamics of those activities [5]. Therefore, it may be promising to identify LBDs from EMG data.

Currently, the exact relationship between LBDs and EMG responses is not clearly known. In [6], a neural network model was proposed to estimate EMG of trunk muscles using kinematics variables. The model was trained by data of healthy subjects and has produced satisfactory results when used to predict EMG of healthy subjects. However, when the

Yanfeng Hou and Jacek M. Zurada are with the Department of Electrical and Computer Engineering, University of Louisville, Louisville, KY 40292, USA (email: y0hou002@louisville.edu, jacek.zurada@louisville.edu).

Waldemar Karwowski is with the Department of Industrial Engineering, University of Louisville, Louisville, KY 40292, USA (email: karwowski@louisville.edu).

William S. Marras is with the Institute for Ergonomics, The Ohio State University, Columbus, OH 43210, USA (email: marras.1@osu.edu). model was used to predict EMG of subjects with low backdisorders, errors of the estimation increased substantially, especially for four of the ten muscles. This indicates that trunk muscle activities of healthy subjects and low backinjured subjects are different, and the difference is reflected on EMG responses. This fact makes it possible to identify LBDs using EMG data of trunk muscles.

Information obtained for evaluation of body stresses in manual lifting activities is often uncertain, imprecise, and noisy [7]. Muscle activities are influenced by multiple factors, without much knowledge of their underlying dynamics. Since the EMG characteristics of trunk muscles of a low back-injured person are unknown, and the differences of EMG responses between a healthy person and an injured person are not fully understood, neural network methods can be used for the LBDs identification purpose.

In this paper, a novel multi-layer feedforward neural network structure with two hidden layers is proposed. The capabilities and characteristics of neural networks with one hidden layer or two hidden layers have been discussed in literature [8]-[13]. In [8], learning capability and storage capacity of two-hidden-layer neural network was studied. In [9], advantages of two-hidden-layer neural network in certain problems were presented. In [10] and [11], classification ability and sensitivity of single hidden-layer neural networks were studied. In this paper, we are not focusing on the differences between single hidden-layer and two-hiddenlayer models. We propose a new structure of neural network with two hidden layers in which certain input variables are connected to the second hidden layer to increase their importance. The proposed network structure is discussed and its effectiveness is validated using benchmark data. The model is then used in the low back-injury identification problem.

Manual materials handling tasks performed in industry have been related to the onset of low back disorders [14]. In this study, we use EMG data collected during manual lifting tasks to build the LBDs identification model. Two methods are discussed in the paper: the 4-muscle method and the 10-muscle method. In both methods, the proposed neural network structure is used. In Section II, the models of those two methods are presented. In Section III, the proposed structure of neural network is discussed and its performance is validated with two benchmark data sets (including both function approximation problem and pattern recognition problem). Simulation results of LBD identification are given in Section IV. 


\section{Two METhods FOR LBD IDENTIFICATION}

To identify low back-injured subjects using EMG signals, ten trunk muscles have been considered: right latissimus dorsi (RLD), left latissimus dorsi (LLD), right erector spine (RES), left erector spine (LES), right rectus abdominus (RRA), left rectus abdominus (LRA), right external oblique (REO), left external oblique (LEO), right internal oblique (RIO), and left internal oblique (LIO). EMG responses of these ten muscles during manual lifting tasks were collected in laboratory. All the EMG data were normalized by maxima recorded from each muscle during a series of six static calibration exertions for the purpose of magnitude comparison and data uncertainty reduction. The normalized EMG data points for each muscle were plotted as a function of normalized time. The details of experimental instruments and data analysis have been reported in [15].

The knowledge about EMG responses of trunk muscles of a low back-injured person is limited. Their characteristics and differences to EMG responses of a healthy person are not fully understood. Since it is not known which features should be extracted from the EMG responses of the trunk muscles for the LBD identification purpose, the original EMG signals are used as input. Such input contains EMG samplings at different stage of a motion to represent the whole motion pattern.

Muscular activities are affected by variety of human- and environment-related factors. Anthropometric characteristics of subjects and task requirements can both affect EMG responses of trunk muscles during a lifting motion. Among those factors, three of task condition variables, such as object weight, lifting height and torso asymmetry are regarded as the most important ones. Therefore, these factors should be taken into account in LBDs identification using EMG signals. In this paper, we only study the sagittal symmetric motions. So only object weight and lifting height are considered (they will be referred to as "task variables" hereafter).

There is no known pattern of EMG response which can tell us if a subject is injured. However, it is known that for subject with LBDs, four trunk muscles (RRA, LRA, REO, and LEO) usually have higher EMG activity level than those observed in healthy people. These four muscles will be referred to as "the four primary muscles" hereafter. For the other six trunk muscles, we do not know if there are differences between injured and non-injured subjects. Therefore, two models are built for LBDs identification, one using EMG of four muscles and the other one using EMG of ten muscles.

\section{A. Method 1: Using EMG Data of Four Muscles}

The first neural network model uses EMG of the four primary muscles. Four neural networks are built for four muscles, one for each respectively. The model diagram is shown in Figure 1. As mentioned above, four trunk muscles of injured subject are often observed to have higher EMG activity level than those four muscles of healthy subject. It is not to say that all the four muscles always follow this rule at the same time. In many cases, at least one of the four muscles has higher EMG activity level. Therefore, if all the neural networks classify the subject as healthy, then the subject is considered as healthy; if one of the neural networks classifies the subject as injured, then the subject is considered as injured. It is a logic "OR" operation between the outputs of the four neural networks.

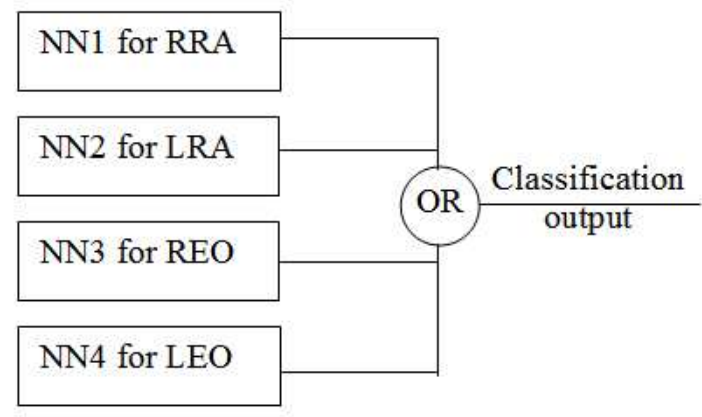

Fig. 1. Diagram of the 4-muscle method

For each of the four neural networks in Figure 1, 30 sampling data points of the corresponding muscle's EMG data of a whole lift motion are used as input, as well as two task variables (object weight and lifting height). As mentioned before, task variables are important because they significantly affect EMG responses of trunk muscles. The same subject can generate totally different EMG responses under different task conditions. Therefore to identify injured subjects using their EMG responses, task variables should be included.

A traditional multilayer feedforward neural network can be constructed using EMG signals of a muscle together with task variables as input, as shown in Figure 2. There are thirtytwo input variables (thirty EMG sampling data points plus two task variables) and one output variable (the classification output). Thirty EMG sampling data points are time series and two task variables are static values. This kind of combined input has a disadvantage that the significance of the task variables can not be shown. According to simulations (will be discussed later), task variables in this configuration are not as effective as expected.

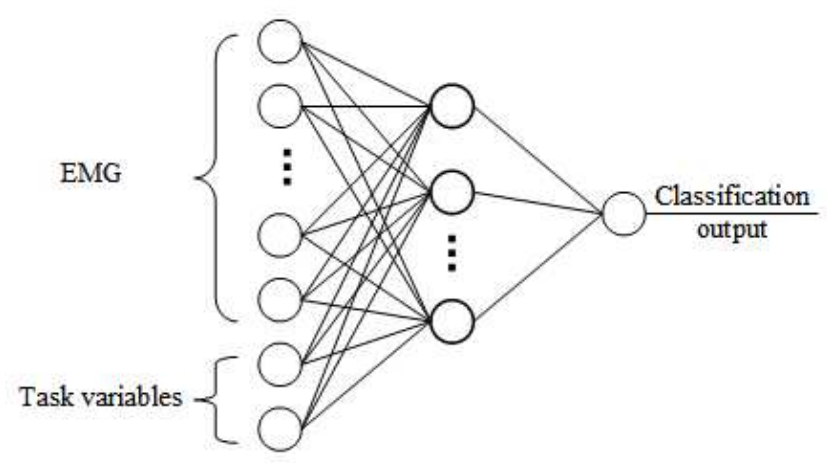

Fig. 2. The traditional network structure for LBDs identification 
To increase the importance of task variables in the network, we consider separating the time series input variables and the static task variables by configuring them in the way shown in Figure 3. Task variables are connected to the second hidden-layer of the network to achieve better performance. The effectiveness of this configuration is proven in Section III and its performance in LBDs identification is discussed in Section IV.

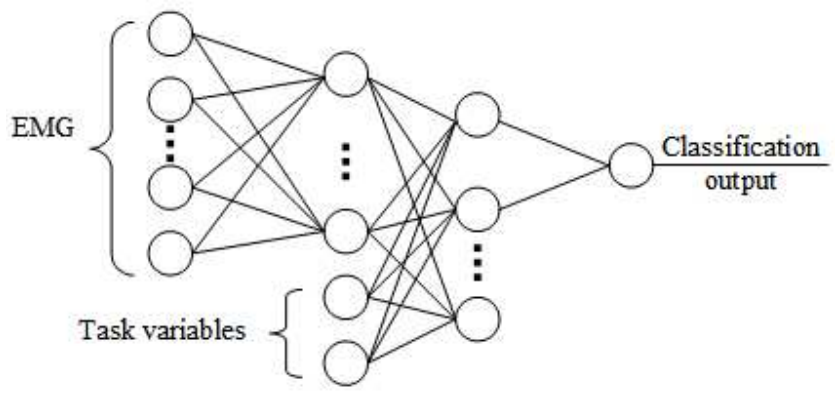

Fig. 3. The proposed network structure for LBDs identification

\section{B. Method 2: Using EMG Data of Ten Muscles}

Using the 4-muscle method, it is expected that in some cases, the model can not correctly identify a subject as healthy or injured. That is because for some subjects with LBDs, none of their four primary muscles show higher EMG activity level than healthy subject; and for some healthy subjects, some of their four primary muscles do occasionally show higher EMG activity level. Thus in these situations, the performance of the model can not be improved if only those four muscles are considered.

Although it is unknown if there are differences in EMG responses of the other six trunk muscles between injured and healthy subjects, the EMG data of those six muscles may provide important information, when considered together with the four primary muscles. It is known that there is a correlation between activities of different muscles. Behavior of the other six muscles can be taken into account if their EMG responses are introduced into the model. Additional information may be provided by those muscles so that decision of classification can be correctly made.

In the 10-muscle method, we can not simply use a logic "OR" operation between outputs of networks of the muscles as we did in Figure 1. The differences in those six muscles' EMG responses between injured and healthy subjects are not known. Their EMG responses do not have the same characteristics as the four primary muscles. Therefore, a way need to be found to make the classification decision based on information of ten muscles' EMG responses. Since a large number of variables are under consideration, a modular neural network model is proposed, as shown in Figure 4.

In modular neural networks [16]-[18], a sub-network is trained independently and in parallel with other subnetworks, using a particular feature subset. The outputs of these sub-networks are mediated by a higher level neural

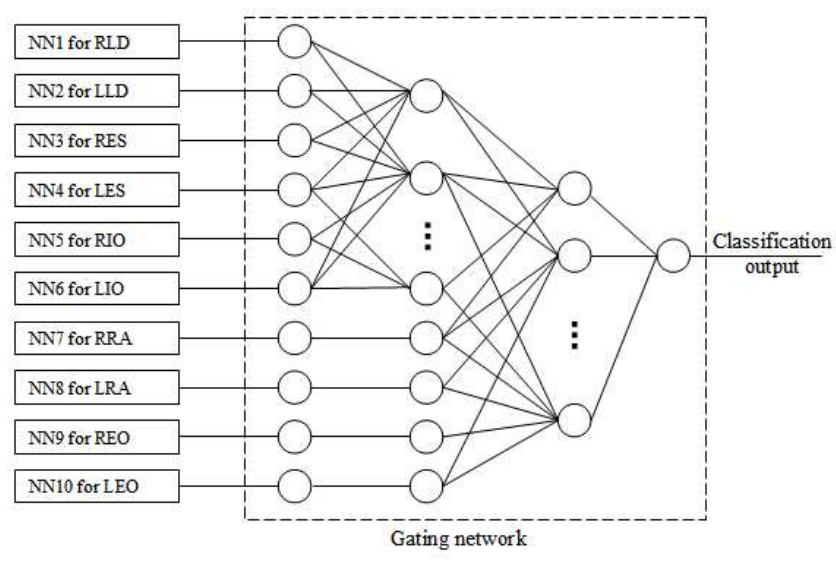

Fig. 4. Diagram of the 10-muscle method

network on the top of the individual networks (a gating network). Modular neural networks have been used in many applications and demonstrated advantages in some aspects over existing methods based on traditional single neural networks [19][20][21]. According to those applications, we can see that the major advantages of modular neural networks are as follows: First, the decomposition of input space makes it easier to deal with complex and large-scale problems. Second, better generalization performance can be achieved because learning subtasks using sub-networks is easier than learning a complex task. Thirdly, the modular neural network is more robust since the failure of one sub-network does not mean that all of the sub-networks fail.

In Figure 4, each of the sub-networks (ten NNs on the left) classifies the subject based on EMG data of one of ten muscles. They are independent neural networks trained offline. The gating network on the right takes outputs of those sub-networks of ten muscles as input and makes the classification decision based on them. This model can take the correlation between muscle activities into account, instead of simply using a logic "OR" operation between outputs of sub-networks.

Again, we configure the gating network in the way as described in the previous subsection: connect outputs of the sub-networks of the four primary muscles to the second hidden-layer since they are more important in LBDs identification. The outputs of the other six networks are used as supplementary information to the gating network. It should be pointed out that if all the muscles are of the same importance, then this configuration has no advantage. This kind of network structure is discussed in the following section.

\section{ALGORITHM AND VALIDATION}

To increase the importance of task variables in the neural network, different types of input variables were separated and the static task variables were connected to the second hidden-layer. In this section, training algorithm is presented and two data sets are used to validate the effectiveness of the network structure. 
Error back-propagation algorithm is used to train the network. The output of the first hidden-layer can be expressed as:

$$
y_{(l 1) t}=f^{h 1}\left(\operatorname{net}_{(l 1) t}\right)=f^{h 1}\left(\sum_{i}^{I} v_{(l 1) t i} \cdot x_{i}\right)
$$

in which

(l1) denotes that it is a variable related to the first hiddenlayer.

$f^{h 1}$ is the activation function of the first hidden-layer.

$v_{(l 1) t i}$ is the weight of connection between the $i$ th input layer neuron and the $t$ th first hidden-layer neuron. $t=$ $1,2, \ldots, T$ is the number of the first hidden-layer neurons. $i=1,2, \ldots, I$ is the number of input variables.

$x_{i}$ is the $i$ th input variable.

The output of the second hidden-layer can be expressed as:

$$
\begin{aligned}
y_{(l 2) p} & =f^{h 2}\left(\text { net }_{(l 2) p}\right) \\
& =f^{h 2}\left(\sum_{t}^{T} v_{(l 2) p t} \cdot y_{(l 1) t}+\sum_{k}^{K} v_{(l 2) p k} \cdot x_{k}^{\prime}\right)
\end{aligned}
$$

in which

(l2) denotes that it is a variable related to the second hidden-layer.

$f^{h 2}$ is the activation function of the second hidden-layer.

$v_{(l 2) p t}$ is the weight of connection between the $t$ th first hidden-layer neuron and the $p$ th second hidden-layer neuron. $p=1,2, \ldots, P$ is the number of the second hidden-layer neurons.

$v_{(l 2) p k}$ is the weight of connection between the $k$ th input neuron (which is connected to the second hidden-layer) and the $p$ th second hidden-layer neuron. $k=1,2, \ldots, K$ is the number of variables which are connected directly to the second hidden-layer.

$x_{k}^{\prime}$ is the $k$ th input variable which is connected to the second hidden-layer.

The output of the output-layer can be expressed as:

$$
o_{q}=f^{o}\left(\operatorname{net}_{q}\right)=f^{o}\left(\sum_{p}^{P} w_{q p} \cdot y_{(l 2) p}\right)
$$

in which

$f^{o}$ is the activation function of the output layer.

$w_{q p}$ is the weight of connection between the $p$ th second hidden-layer neuron and the $q$ th output-layer neuron.

The error function to be minimized is

$$
E=\frac{1}{2} \sum_{k=1}^{K}\left(d_{q}-o_{q}\right)^{2}
$$

where $d_{q}$ and $o_{q}$ are the actual output and the network output, respectively.
Weight adjustment of $w_{q p}$ is calculated as:

$$
\Delta w_{q p}=\eta \cdot\left(d_{q}-o_{q}\right) \cdot \frac{\partial f^{o}\left(\text { net }_{q}\right)}{\partial n e t_{q}} \cdot y_{(l 2) p}
$$

where $\eta$ is the learning rate.

Let

$$
\operatorname{Odel}_{q}=\left(d_{q}-o_{q}\right) \cdot \frac{\partial f^{o}\left(\text { net }_{q}\right)}{\partial n e t_{q}}
$$

Weight adjustment of $v_{(l 2) p j}$ can be calculated as:

$$
\Delta v_{(l 2) p j}=\eta \cdot \sum_{q}^{Q} w_{q p} \cdot \operatorname{Odel}_{q} \cdot \frac{\partial f^{h 2}\left(\operatorname{net}_{(l 2) p}\right)}{\partial n e t_{(l 2) p}} \cdot y_{(l 1) j}
$$

in which $j=1,2, \ldots, J$ and $J=T+K$.

If we let

$$
\operatorname{Hdel}_{p}=\sum_{q}^{Q} w_{q p} \cdot \operatorname{Odel}_{q} \cdot \frac{\partial f^{h 2}\left(\text { net }_{p}\right)}{\partial n e t_{p}},
$$

weight adjustment of $v_{(l 1) t i}$ can be written as:

$$
\Delta v_{(l 1) t i}=\eta \cdot \sum_{p}^{P} v_{(l 2) p t} \cdot \operatorname{Hed}_{p} \cdot \frac{\partial f^{h 1}\left(\operatorname{net}_{(l 1) t}\right)}{\partial n e t_{(l 1) t}} \cdot x_{i}
$$

To investigate the influence of inputs connected to the first hidden-layer and inputs connected to the second layer, we use the configuration shown in Figure 5. In Figure 5 (A), the effective input (real input) is connected to the first hidden-layer, and random signals (dummy input) of the same size of the effective input is connected to the second hiddenlayer. In Figure 5 (B), the locations of real input and dummy input are exchanged. By examining the performance of these two networks, we can find out the importance of inputs at different places.

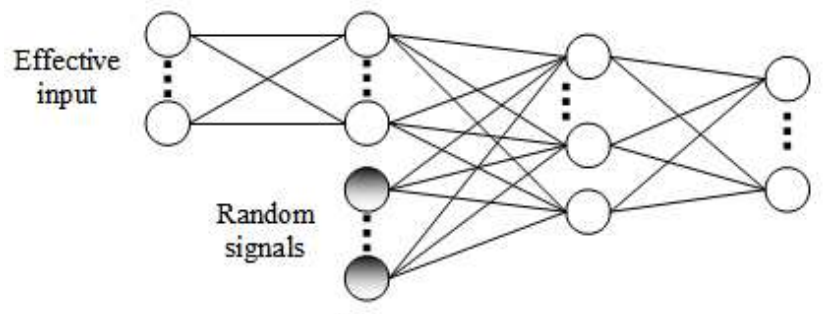

(A)

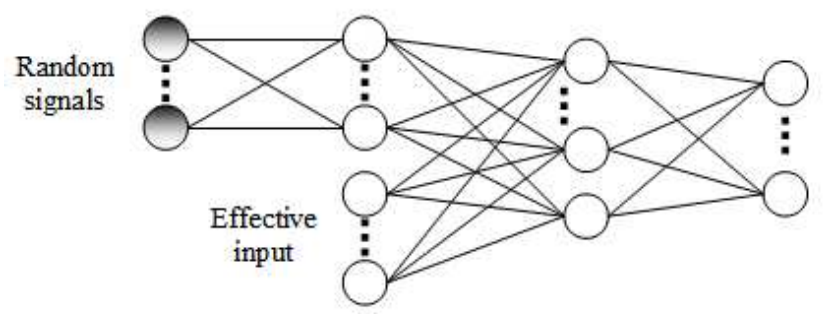

(B)

Fig. 5. Comparing importance of inputs to the first hidden-layer and to the second hidden-layer. A) Effective input is connected to the first hidden-layer. B) Effective input is connected to the second hidden-layer. 


\section{A. Example 1: A Function Approximation Problem}

The first experiment is carried out with the automobile fuel consumption prediction problem. In this problem, variables such as model year and horsepower are used to predict miles per gallon (MPG) of the automobiles. Data set used here is from [23]. It consists of 392 complete instances without missing values. Each instance is composed of six input variables (number of cylinders, displacement, horsepower, weight, acceleration, and model year) and one output variable (MPG). Random input signals (dummy input signals) are generated using Matlab command with the same size of the real input pattern set.

Simulations are done on the two configurations shown in Figure 5 (A) and Figure 5 (B), using the same network parameters: 6 inputs to the first hidden-layer (real input or dummy input), 6 inputs to the second hidden-layer (dummy input or real input), 1 output, 300 training patterns, 92 test patterns, 16 neurons in the first hidden-layer, 18 neurons in the second hidden-layer, learning rate 0.6. The same random input signals are used. Training profile of the two networks are shown in Figure 6. We can find that training error of the second network drops faster than training error of the first network, which means the second one converges faster than the first one.
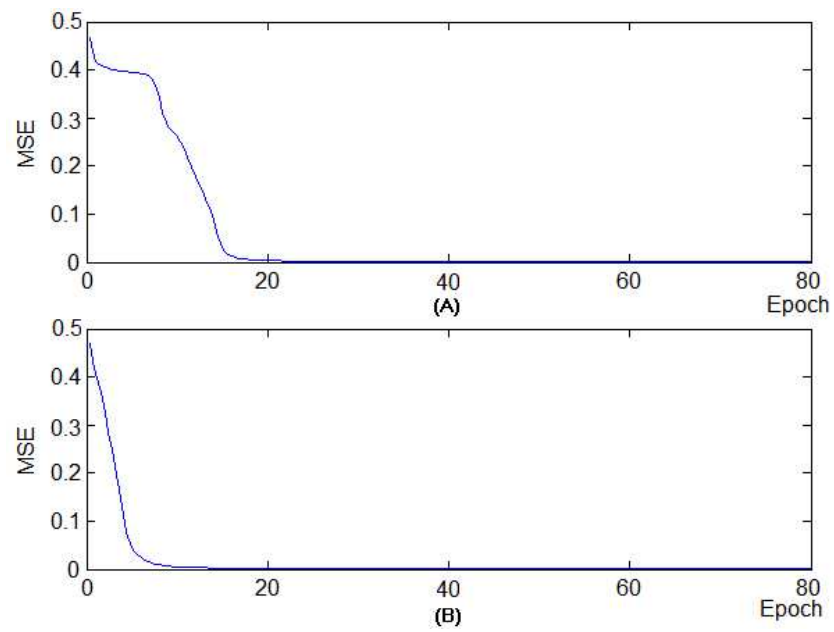

Fig. 6. Training error of the fuel consumption prediction problem. A) Using network in Figure 5 (A). B) Using network in Figure 5 (B).

To guard against bias caused by different random input signals and random initial weight values, every simulation was performed five times. The mean absolute errors (MAE) of the output of all simulations are listed in Table I. It is obvious that MAE of the network in Figure 5 (B) are smaller than MAE of the network in Figure 5 (A). Therefore it confirms that input to the second hidden-layer has more influence than input to the first hidden-layer.

\section{B. Example 2: A Pattern Recognition Problem}

The second experiment is carried out with the digit recognition problem. In this problem, images of hand-drawn digits need to be recognized by the neural network. The images are
TABLE I

MeAn AbSOLUte ERrors of THE FUEL CONSUMPTION PREDICTION

PROBLEM

\begin{tabular}{|c|c|c|}
\hline Simulations & MAE using Fig. 5 (A) & MAE using Fig. 5 (B) \\
\hline 1 & 0.1019 & 0.0697 \\
\hline 2 & 0.1002 & 0.0863 \\
\hline 3 & 0.0954 & 0.0565 \\
\hline 4 & 0.1024 & 0.0584 \\
\hline 5 & 0.1269 & 0.0585 \\
\hline Average & 0.1054 & 0.0659 \\
\hline
\end{tabular}

vectors of 20 numbers representing 5-by-4 pixel gray-level images, with gray levels normalized in the range 0 to 1 . These vectors stand for four digits: zero, one, two, and three.

Again, simulations are done on the two networks shown in Figure 5 (A) and Figure 5 (B), using the same network parameters: 20 inputs to the first hidden-layer (real input or dummy input), 20 inputs to the second hidden-layer (dummy input or real input), 4 outputs, 32 training patterns, 8 test patterns, 8 neurons in the first hidden-layer, 8 neurons in the second hidden-layer, learning rate 0.6. Training errors of the two networks are shown in Figure 7. Similar to Example 1, training error of the second network drops faster than training error of the first network.
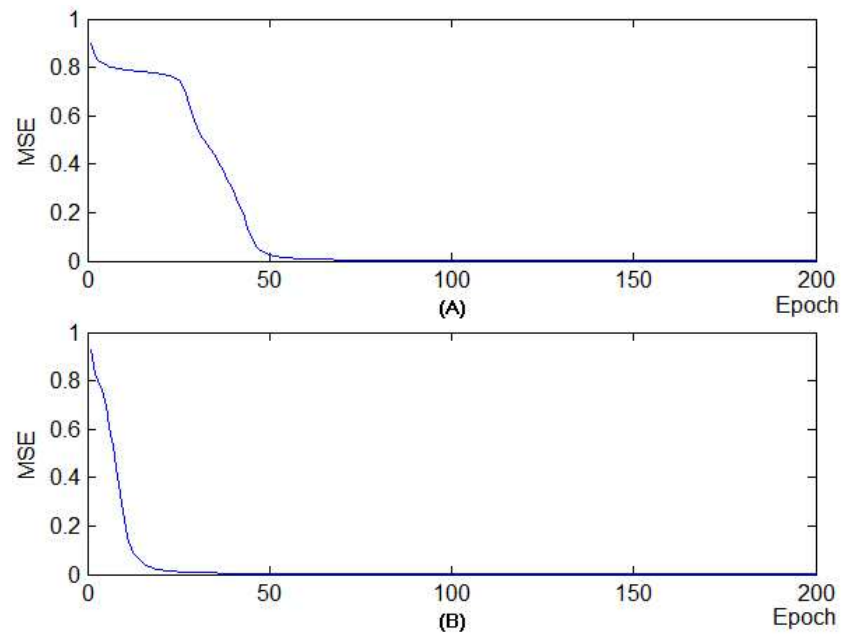

Fig. 7. Training error of the digit recognition problem. A) Using network in Figure 5 (A). B) Using network in Figure 5 (B).

Simulations were also performed five times. Mean absolute errors of the output of all simulations are listed in Table I. As we can see, MAE of the network in Figure 5 (B) are also smaller than MAE of the network in Figure 5 (A). This again confirms that input to the second hidden-layer has more influence than input to the first hidden-layer. Therefore this structure is used in LBDs identification problem to make the task variables more effective. 
TABLE II

MeAn Absolute ERror of THE Digit RECOGNITION PROBLEM

\begin{tabular}{|c|c|c|}
\hline Simulations & MAE using Fig. 5 (A) & MAE using Fig. 5 (B) \\
\hline 1 & 0.1787 & 0.1136 \\
\hline 2 & 0.1915 & 0.1145 \\
\hline 3 & 0.1814 & 0.1225 \\
\hline 4 & 0.1834 & 0.1160 \\
\hline 5 & 0.1819 & 0.1124 \\
\hline Average & 0.1834 & 0.1158 \\
\hline
\end{tabular}

\section{Simulations and Results}

\section{A. The Data Set and Its Preprocessing}

The data set used for LBDs identification contains 44 subjects, in which half of them (22) have low back disorders. EMG responses of ten trunk muscles (RLD, LLD, RES, LES, RRA, LRA, REO, LEO, RIO, and LIO) of 1588 trials were recorded. All of these trials are sagittal symmetrical motions. EMG data were normalized by maxima recorded from each muscle during a series of six static calibration exertions. The normalized EMG data points for each muscle were plotted as a function of normalized time. Task conditions are defined by the combination of three factors: object weight, original height, and destination height. Lifting height can be obtained by using the destination height minus the original height. Object weight includes 10 pounds, 15 pounds, 20 pounds, and 25 pounds. Original height includes floor, knee, waist, and shoulder. Destination height is fixed at elbow. Except from shoulder to elbow (which is a lowering motion), the other conditions are lifting motions (from floor to elbow, from knee to elbow, and from waist to elbow).

EMG data and task variables are preprocessed to avoid illconditioning before they can be used in neural network model [6]. For EMG data, three procedures are performed. They are synchronizing, re-sampling and re-scaling. EMG data of all muscles and of all subjects are synchronized to represent the whole process of a motion so that incorrect timing can be avoided. At the same sampling rate, different subjects might have different number of sampling points if the motion speed is different. Since the input dimension of the model is constant, re-sampling is needed to make sure all the motions have the same number of EMG sampling points. The original measurements of task variables are not suitable for neural networks. Thus re-scaling these variables is necessary. These preprocessing procedures are done in computer programs.

\section{B. Results}

Conservative cross-validation method is used in the simulations. Every time EMG data of all participated trials of two injured subjects and two healthy subjects were taken out as test data. Data of all other subjects were used to train the network. Performance of different models for LBDs identification is compared below.
TABLE III

PARAMETERS AND RESULTS OF THE TRADITIONAL STRUCTURE AND THE PROPOSED STRUCTURE FOR 4-MUSCLE METHOD

\begin{tabular}{|c|c|c|}
\hline Parameters & Traditional Structure & Proposed Structure \\
\hline Number of inputs & 32 & $30+2$ \\
\hline Number of outputs & 1 & 1 \\
\hline Hidden layers & 1 & 2 \\
\hline Hidden neurons & 15 & $10+10$ \\
\hline Learning rate & 0.015 & 0.015 \\
\hline Accuracies $(\%)$ & 78.3 & 81.4 \\
\hline
\end{tabular}

1) Performance of the Traditional Structure and the Proposed Structure in 4-muscle Method: In the 4-muscle method, two network structures were presented in Section 2. The traditional network structure (Figure 2) and the proposed network structure (Figure 3) were both used for the four neural networks in Figure 1. Simulations were done for both networks to obtain comparison results.

In the traditional network structure, thirty EMG inputs and two task variable inputs are combined as a single input vector. Using this structure, an accuracy of $78.3 \%$ was obtained for LBDs identification (1243 successful classifications over 1588 trials total). To increase the importance of task variables in the network, the EMG input (time series) and the task variables (static values) are separated by connecting task variables to the second hidden-layer of the network. Table III shows that better performance has been achieved. Parameters in the table are for each of the four networks NN1 to NN4 in Figure 1 (in the expression " $30+2$ " and " $10+10$ ", the first number is parameter of the first hidden-layer, and the second number is the parameter of the second hidden-layer).

The errors of misclassification include two conditions:

- 1) a subject was classified incorrectly in all participated task conditions;

- 2) a subject was classified incorrectly in some participated task conditions.

Most unsuccessful cases belong to the first condition. This means if in one task condition a subject is classified as injured (or healthy) by the model, then in other task conditions he is often also classified as injured (or healthy). There are exceptions that a subject is classified correctly in some task conditions but he is classified incorrectly in other task conditions. As stated before, the same subject can have very different EMG responses for different task conditions. Given this fact, the performance of the model is consistent through all task conditions. That is because task variables are used as input variables to the model. Less than $10 \%$ exceptions that occurred when the proposed network structure was used. Compared to about $20 \%$ of exceptions happened when the traditional network structure was used, the proposed network structure has improved the importance of task variables. 
TABLE IV

ClassificAtion RESUlts OF 4-MUSCLE METHOD AND 10-MUSCLE METHOD

\begin{tabular}{|c|c|}
\hline LBD trials & 788 \\
\hline Non-LBD trials & 800 \\
\hline Total trials & 1588 \\
\hline 4-muscle method correct trials & 1293 \\
\hline 4-muscle method accuracy (\%) & 81.4 \\
\hline 10-muscle method correct trials & 1412 \\
\hline 10-muscle method accuracy (\%) & 88.9 \\
\hline
\end{tabular}

TABLE V

CLASSIFICATION ACCURACIES OF DIFFERENT OBJECT WEIGHTS

\begin{tabular}{|c|c|c|c|c|}
\hline Conditions & 1 & 2 & 3 & 4 \\
\hline Object weight (lbs.) & 10 & 15 & 20 & 25 \\
\hline LBD trials & 234 & 218 & 194 & 143 \\
\hline Non-LBD trials & 230 & 222 & 200 & 147 \\
\hline Total trials & 464 & 440 & 394 & 290 \\
\hline Correct trials & 421 & 398 & 344 & 250 \\
\hline Accuracies $(\%)$ & 90.7 & 90.5 & 87.3 & 86.2 \\
\hline
\end{tabular}

2) Performance of the 4-Muscle Method and the 10Muscle Method: Performance of the 4-muscle method and the 10-muscle method is also compared. Table IV shows number of successfully identified trials and classification accuracies of the two methods. Benefited from more information provided (not only EMG data of the other six muscles but also the underlying correlation between muscle activities), the 10-muscle method can correctly identify more cases than the 4-muscle method.

Parameters of the gating network in the 10-muscle method are as follows: six input variables to the first hidden-layer; four input variables to the second; one output variable in the output layer; eight neurons in the first; twelve neurons in the second; learning rate is 0.1 . The number of hidden neurons is decided using a trial-and-error procedure. The final LBDs identification accuracy of this model is $88.9 \%$. The 10-muscle method using a modular neural network model has exhibited better performance than the 4-muscle method which uses a simple logic "OR" operation between outputs of different networks.

3) More Results: To improve understanding of EMG responses of subjects with low back injuries under different task conditions, LBDs identification accuracies of different object weight are calculated and listed in Table V. For object weight of 10 pounds, 15 pounds, 20 pounds and 25 pounds, we found that the LBDs identification accuracies are actually decreasing when the weight is increasing.

This is an interesting finding. Naturally, we hypothesized that when the object weight increases, the difference of EMG responses between injured subjects and healthy subjects
TABLE VI

CLASSIFICATION ACCURACIES OF DIFFERENT LIFTING HEIGHTS

\begin{tabular}{|c|c|c|c|c|}
\hline Conditions & 1 & 2 & 3 & 4 \\
\hline Motion type & Lowering & Lifting & Lifting & Lifting \\
\hline Orig. height & shoulder & Waist & Knee & Floor \\
\hline Dest. height & Elbow & Elbow & Elbow & Elbow \\
\hline Avg. abs. height $(\mathrm{cm})$ & 36.0 & 15.3 & 57.8 & 110.5 \\
\hline LBD trials & 141 & 276 & 259 & 113 \\
\hline Non-LBD trials & 150 & 262 & 252 & 135 \\
\hline Total trials & 291 & 538 & 511 & 248 \\
\hline Correct trials & 266 & 480 & 452 & 214 \\
\hline Accuracies $(\%)$ & 91.6 & 89.1 & 88.5 & 86.1 \\
\hline
\end{tabular}

would be more significant. Consequently the classification would be easier to perform. However, results in Table V show that it may not be true. It seems when the object weight is greater (which means more muscle exertion is needed), EMG responses of injured and non-injured subjects behave more similarly. To obtain more evidence and validate this new finding, LBDs classification accuracies of different lifting heights were calculated, as shown in Table VI.

In Table VI, motion type, original height, destination height, absolute height (average), number of successfully identified trials and classification accuracies are listed. The motion from shoulder to elbow is a lowering motion which requires less muscle exertion than the other lifting motions in the table. Correspondingly, we found that its LBDs classification accuracy is the highest. As for the lifting motions, it is also observed that the higher lifting height (more muscle exertion), the lower LBDs classification accuracy. Therefore according to the results given in and Table V and Table VI, LBDs classification accuracy decreases when object weight or lifting height increase. Does this mean when object weight or lifting height increases, muscles have to be recruited on a high (and similar) activity level, no matter if the subject is injured or healthy? The obtained results increase our understanding of low back-disorders and may provide important guidance for future experimental studies.

\section{CONCLUSIONS AND Discussions}

In this paper we discussed a novel neural network structure and its application in the identification of low back disorders using EMG data. Benchmark data validation for the proposed neural network was provided. A 4-muscle method and a 10muscle method are discussed. Higher classification accuracy was achieved by the 10-muscle method. It was found that the LBDs identification accuracy decreases when object weight or lifting height increase.

EMG responses of trunk muscles under different task conditions such as different object weight and different lifting height were studied. However, different torso asymmetries were not involved in this research. Such a study could be done to gain more knowledge about EMG responses of low back-injured subjects. 
Considering the complexity of human muscle activities and the differences of these activities between subjects, the performance of the developed models may be further improved if data sets of more subjects are used. Also, conservative cross-validation method was applied in the simulations. Evaluating performance of the LBDs identification model on independent data sets would be desirable.

According to the performance of the proposed model, it would be promising to estimate the extent of a disorder using EMG data, if related information is provided. This application can be of great importance since currently few quantitative methods can effectively identify the extent of LBDs.

\section{ACKNOWLEDGMENT}

This study was conducted under a research grant on the "Development of a Neuro-Fuzzy System to Predict Spinal Loading as a Function of Multiple Dimensions of Risk", sponsored by the National Institute for Occupational Safety and Health (DHHS).

\section{REFERENCES}

[1] W.S. Marras, S.A. Ferguson, P. Gupta, S. Bose, M. Parnianpour, J.Y. Kim, R.R. Crowell, "The quantification of low back disorder using motion measures", Spine, 1999, vol. 24, no. 20, pp. 2091 - 2099

[2] S. Bigos, M. Battiee, "The impact of spinal disorders in industry", The adult spine: principles and practice, 2nd ed. Frymoyer, JW, ed., Philadelphia: Lippincott-Taven, 1997: 151-61

[3] W.S. Marras, K.E.K. Lewis, S.A. Ferguson, M. Parnianpour, "Impairment magnificaiton during dynamic trunk motions", Spine, 2000, vol. 25 , no. 5, pp. 587 - 595

[4] W.S. Marras, M. Parnianpour, S.A. Ferguson, J.Y. Kim, R.R. Crowell, S. Bose, S.R. Simon, "The classification of anatomic- and syptom-based low back disorders using motion measure models", Spine, 1995, vol. 20, no. 23 , pp. $2531-2546$

[5] P.A. Crosby, "Use of surface electromyogram as a measure of dynamic force in human limb muscles", Med. and Biol. Eng. and Comput., vol. 16, 1978, pp. 519-524.

[6] Y. Hou, J.M. Zurada, W. Karwowski, "Prediction of EMG signals of trunk muscles in manual lifting using a neural network model", Proc. of the International Joint Conference on Neural Networks (IJCNN 2004), Budapest, Hungary, Jul. 25-29, 2004, pp. 1935-1940

[7] W. Karwowski, "The Human World of Fuzziness, Human Entropy, and the Need for General Fuzzy Systems Theory", Journal of Japan Society for Fuzzy Theory and Systems, 1992, vol. 4 no. 5, pp. 591-609

[8] G.B. Huang, "Learning capability and storage capacity of two-hiddenlayer feedforward networks", IEEE Transactions on Neural Networks, March, 2003, vol. 14, no. 2, pp. $274-281$

[9] E.D. Sontag, "Feedback stabilization using two-hidden-layer nets", IEEE Transactions on Neural Networks, Nov. 1992, vol. 3, no. 6, pp. $981-990$

[10] G.B. Huang; Y.Q. Chen, H.A. Babri, "Classification ability of single hidden layer feedforward neural networks", IEEE Transactions on Neural Networks, May 2000, vol. 11, no. 3, pp. 799 - 801

[11] S.H. Oh; Y. Lee, "Sensitivity analysis of single hidden-layer neural networks with threshold functions", IEEE Transactions on Neural Networks, July 1995, vol. 6, no. 4, pp. $1005-1007$

[12] J. de Villiers, E. Barnard, "Backpropagation neural nets with one and two hidden layers", IEEE Transactions on Neural Networks, vol. 4, no. 1, Jan. 1993 pp. $136-141$

[13] S.H. Oh, S.Y. Lee, "A new error function at hidden layers for past training of multilayer perceptrons", IEEE Transactions on Neural Networks, vol. 10, no. 4, July 1999 pp. $960-964$

[14] W. Karwowski, W.S. Marras (Editors), "The Occupational Ergonomics Handbook", 1999, CRC Press, Boca Raton.

[15] W.S. Marras, K.P. Granata, "Spine loading during trunk lateral bending modtions", J Biomech, 1997, vol. 30, no. 7, pp. 697 - 703

[16] L. Wang; S.Z. Der, N.M. Nasrabadi, "Automatic target recognition using a feature-decomposition and data-decomposition modular neural network", IEEE Transactions on Image Processing, vol. 7, no. 8, Aug. 1998 pp. $1113-1121$

[17] T. Hong; M.T.C. Fang, "Detection and classification of partial discharge using a feature decomposition-based modular neural network", IEEE Transactions on Instrumentation and Measurement, vol. 50, no. 5, Oct. 2001 pp. 1349 - 1354

[18] R. Anand, K. Mehrotra, C.K. Mohan, S. Ranka, "Efficient classification for multiclass problems using modular neural networks", IEEE Transactions on Neural Networks, vol. 6, no. 1, Jan. 1995 pp. 117 124

[19] A. Khotanzad, R.C. Hwang; A. Abaye, D. Maratukulam, "An adaptive modular artificial neural network hourly load forecaster and its implementation at electric utilities", IEEE Transactions on Power Systems, vol. 10, no. 3, Aug. 1995 pp. 1716 - 1722

[20] B.L. Lu, J. Shin, M. Ichikawa, "Massively parallel classification of single-trial EEG signals using a min-max Modular neural network", it IEEE Transactions on Biomedical Engineering, vol. 51, no. 3, March 2004 pp. $551-558$

[21] R. A. Jacobs, M. I. Jordan, "Learning piecewise control strategies in a modular neural network architecture", it IEEE Transactions on Systems, Man and Cybernetics, vol. 23, no. 2, March-April 1993 Ppp. 337 - 345

[22] J.M. Zurada, Introduction to Artificial Neural Systems, PWS Publishing Company, 1992

[23] UCI Machine Learning Repository, http://www.ics.uci.edu/ mlearn/ MLRepository.html 